\title{
A visualization and computational study of horizontal Bridgman crystal growth
}

\author{
C.W. $\operatorname{Lan}^{\mathrm{a}, *}$, M.C. $\mathrm{Su}^{\mathrm{b}}$, M.C. Liang ${ }^{\mathrm{b}}$ \\ ${ }^{a}$ Chemical Engineering Department, National Taiwan University, Taipei 106, Taiwan, ROC \\ ${ }^{\mathrm{b}}$ Chemical Engineering Department, National Central University, Chung-Li 32054, Taiwan, ROC
}

Received 12 October 1998; accepted 19 July 1999

Communicated by J.B. Mullin

\begin{abstract}
A transparent three-zone horizontal Bridgman furnace was used for the crystal growth of sodium nitrate. During crystal growth, the heating profiles and the growth front shape as well as flow patterns were recorded. To further understand the heat flow, a three-dimensional computer model based on an efficient multigrid finite volume method was also used to simulate the growth. Key process parameters, including the heating profiles, the growth rate, and the booster heating, were considered and their effects on the interface shape and the flow structures were discussed. It was observed that both buoyancy and Marangoni flows were dominant in the melt causing a highly concave interface. As a result, single-crystal growth was very difficult. Suitable insulation at the furnace bottom reduced interface deflection. Further, interface inversion was obtained by using a booster heating while keeping a cold spot at the top furnace wall near the interface by blowing cooling air. The obtained convex interface shape thus made the growth of a single crystal much easier. (C) 2000 Elsevier Science B.V. All rights reserved.
\end{abstract}

PACS: 44.25. + f; 47.27.Te; 81.10.Fq; 02.60.Cb; 02.70.Fk

Keywords: Visualization; Simulation; Interface shape; Horizontal Bridgman; Convection

\section{Introduction}

The horizontal Bridgman (HB) technique is an important crystal growth method. Because the top surface of the material is not confined to the crucible, the defects due to the crucible contact can be greatly reduced. The removal of the grown crystal is also easier as compared with the vertical configuration. Therefore, the HB method has become one

\footnotetext{
* Corresponding author. Fax: + 886-2-2363-3917.

E-mail address: lan@ruby.che.ntu.edu.tw (C.W. Lan)
}

of the major processes for the growth of III-V compound semiconductor bulk crystals [1]. Recently, this method has also been used for the growth of oxide laser crystals [2]. Like other crystal growth processes, the control of the growth interface is crucial to the $\mathrm{HB}$ growth. A concave interface often induces parasitic nucleation on the crucible wall leading to a polycrystalline growth. However, interface control is not a trivial task and often requires a detailed understanding of the heat transfer and fluid flow during crystal growth. As compared with other crystal growth processes, the heat flow in HB growth is also more complicated. 
In addition to thermocapillary and buoyancy flows, the problem is three-dimensional (3D) and often time-dependent in nature. In the present paper, we attempt to use growth visualization and computer simulation to understand the heat flow and its effects on the growth front during crystal growth. Furthermore, simple ideas to control the growth front will be proposed and tested.

Computer simulation is a cheaper way to provide a quick understanding of the process. Many two-dimensional (2D) computations were conducted for HB crystal growth in the past decade (e.g., Refs. [3-6]). The coupled solution of heat flow and growth interface has also been considered [4-6]. However, 2D modeling is not realistic to HB crystal growth. Recently, some effort has been made for modeling 3D flows in HB growth [7-9]. In most of the computation, the growth interface was assumed fixed or the thermocapillary convection was not considered. A more complete study was carried out by Liang and Lan [10] recently. They developed a 3D HB model using a front tracking technique based on a finite volume method (FVM) to simulate the $\mathrm{HB}$ growth of GaAs. Both buoyancy and thermocapillary flows and their effects on the growth interface were considered. However, the solution was based on a single-grid iterative approach, and the convergence rate was slow. To improve the convergence, a multigrid (MG) version of their model was then developed [11]. In this paper, the newly developed method will be used to simulate the $\mathrm{HB}$ growth of sodium nitrate $\left(\mathrm{NaNO}_{3}\right)$.

Besides the modeling, visualization is an efficient way to understand the growth. For the HB growth, not much work has been reported for in situ flow and interface visualization. The experimental results by Yao and Groh [8] and Yeoh et al. [9] for the HB growth of succinonitrile (SCN) are the only results reported. However, their experiments were performed in a closed ampoule, in which no free surface existed. Therefore, only natural convection was considered in their study. The coupling of thermocapillary and buoyancy flows as well as their individual effects was considered by Schwabe and Metzger [12] for a rectangular geometry (the aspect ratio is less than one). However, their configurations were not realistic for the $\mathrm{HB}$ growth; the effects of flows on the growth interface were not considered. Therefore, a more realistic way is to conduct a real growth experiment using a transparent system, while recording the flow patterns and interface shape simultaneously. Such a study may provide useful information for crystal growers to control the growth interface better.

In this study, we presented a transparent system for the HB growth of sodium nitrate. Sodium nitrate is a good candidate for growth visualization since its melt and crystal are transparent. The lower melting point $\left(306.8^{\circ} \mathrm{C}\right)$ also makes the experiment setup simpler. Since sodium nitrate is a high Prandtl-number material $(\mathrm{Pr}=9.2)$, the effects of flows are expected to be significant. Its thermal expansion and surface-tension-temperature coefficients are also large enough to induce significant convection. In addition to the flow and interface observation, 3D computer simulation is also conducted to study the growth, especially the heat transfer. Furthermore, the main purpose of understanding heat transfer and fluid flow is to control the growth interface, which is crucial to grow single crystals. Therefore, in addition to illustrating the roles of heat flow, we will also discuss the ways for better interface control. In the next section, the experimental setup and procedures are described, followed by a brief description of computer simulation in Section 3. Section 4 is devoted to the results and discussion. Short conclusions are given in Section 5 .

\section{Experimental procedure}

The construction of the transparent three-zone $\mathrm{HB}$ furnace is sketched in Fig. 1. The furnace was made of a Pyrex glass tube $(\mathrm{ID}=4.6 \mathrm{~cm}, \mathrm{OD}=$ $5.0 \mathrm{~cm}$, and length $=45 \mathrm{~cm}$ ) with three heaters. Each heater was made of a $0.65 \mathrm{~mm}$ diameter Nichrome wire. The hot and cold zones had the same length (about $21 \mathrm{~cm}$ long), while the center zone (for the booster heater) was only $1.5 \mathrm{~cm}$ in length. Both ends of the hot and cold zones also had $10 \mathrm{~cm}$ insulation to maintain a constant temperature there. The power of the three zones were controlled independently. During crystal growth, the temperature distributions of the inner wall of the furnace 


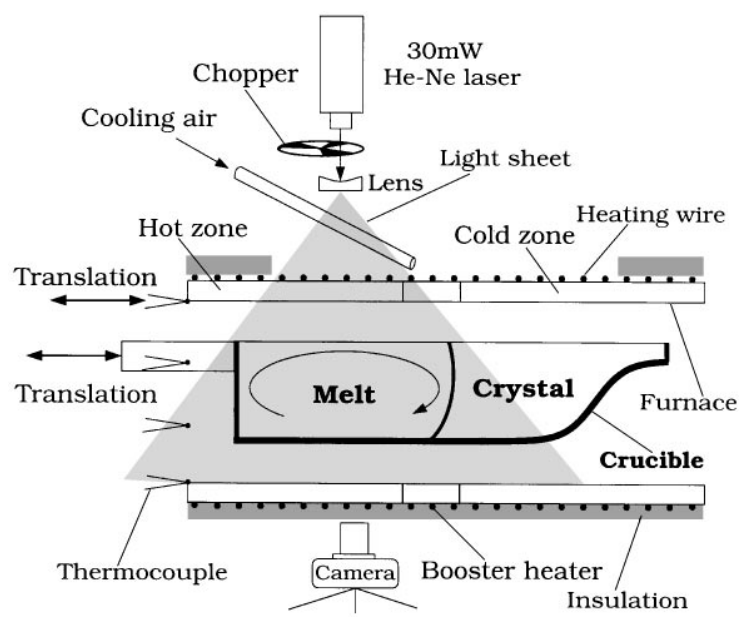

Fig. 1. A schematic of experimental setup for horizontal Bridgman crystal growth of sodium nitrate.

were measured. Since the thermal distributions were not axisymmetric, eight K-type thermocouples $\left(0.5 \mathrm{~mm}\right.$ in diameter) with $45^{\circ}$ apart were used to measure the axial temperature profiles at different points of the cross section for a given distance. The translation of the thermocouples were controlled by a microstepping motor. Insulation could be added at the bottom or the two side walls of the furnace if necessary. However, due to the natural convection of the air, the top wall of the furnace was always hotter than the bottom if no insulation was used. Such a configuration resulted in an extremely deformed interface shape and made single-crystal growth difficult.

The crucible for the growth was made of a half (about) of a Pyrex glass tube. The body part had $\mathrm{ID}=2.8 \mathrm{~cm}, \mathrm{OD}=3.2 \mathrm{~cm}$, and length $=12 \mathrm{~cm}$, while the seeding part had $\mathrm{ID}=0.8 \mathrm{~cm}, \mathrm{OD}=$ $1.2 \mathrm{~cm}$, and length $=1.5 \mathrm{~cm}$. The end of the crucible was connected to a Pyre glass rod to hold it in a suitable position (about at the center part of the furnace). The rod was connected to a high precision translation system. Crystal growth rate was then controlled by a microstepping motor through the translation system. To start the growth, sodium nitrate powder was fed into the crucible located in the hot zone. The amount of the powder was carefully adjusted so that the crucible was filled with the melt exactly. After the system was stabilized the crucible was moved towards the cold zone for crystal growth; no seed was used. For the cases with a flat or convex growth front, the solidified sodium nitrate had only a few grains at the beginning, and a single grain can be selected quickly after about $1-2 \mathrm{~cm}$ growth distance. However, if the interface was concave, polycrystalline growth was always obtained.

After the growth interface was about in the middle of the crucible, both temperature profiles and the interface shape (both top and side views) were recorded. To visualize the flow, a small amount of aluminum particles ( $20 \mu \mathrm{m}$ in diameter) was used as a tracer for flow visualization. A $30 \mathrm{~mW} \mathrm{He-Ne} \mathrm{laser} \mathrm{(Meredith} \mathrm{Instruments} \mathrm{Inc.)}$ was used as a light source. After the laser beam passed through a cylindrical lens, a light sheet with $8 \mathrm{~cm}$ in width and $0.5 \mathrm{~mm}$ in thickness was produced. To measure the particle velocity, a chopper (Oriel Inc.) was also used. However, the particle velocity near the free surface was hard to measure. Therefore, we can report the velocity values near the free surface for reference only.

\section{Computer simulation}

A 3D model was also developed to simulate the growth experiments. The flow observation indicated a steady flow; oscillatory flow was not observed. Therefore, a pseudo-steady state was assumed. Furthermore, the interface shape did not change much near the middle of the growth; it changed more significantly near seeding and shouldering stages. Also, the flow was assumed laminar, and the melt was further assumed to be Newtonian and incompressible; the body force was considered through the Boussinesq approximation. Furthermore, the free surface was assumed to be flat and rigid; the deformation of the free surface due to the flow and the growth angle (less than $2.5^{\circ}$ ) was also small. During growth, the melt level was kept as same as the crucible top edges, so that the flatsurface assumption was reasonable. With the assumptions, the governing equations were setup. The measured profiles were also used as the input for simulation. However, heat transfer coefficients were adjusted to fit the observed interface shape. 
Table 1

Physical properties of $\mathrm{NaNO}_{3}$ and crucible [13]

$\mathrm{NaNO}_{3}$

$T_{\mathrm{m}}=306.8^{\circ} \mathrm{C}$

$\Delta H=182 \mathrm{~J} \mathrm{~g}^{-1}$

$h=4.6 \times 10^{-4} \mathrm{~W} \mathrm{~cm}^{-2}{ }^{\circ} \mathrm{C}^{-1}$

$k_{\mathrm{S}}=5.65 \times 10^{-3}-33.5(T-230) \times 10^{-7} \mathrm{~W} \mathrm{~cm}^{-1}{ }^{\circ} \mathrm{C}^{-1}$

$k_{\mathrm{L}}=5.65 \times 10^{-3}+44.7\left(T-T_{\mathrm{m}}\right) \times 10^{-7} \mathrm{~W} \mathrm{~cm}^{-1}{ }^{\circ} \mathrm{C}^{-1}$

$C p_{\mathrm{S}}=C p_{\mathrm{L}}=1.255+2.18(T-100) \times 10^{-3} \mathrm{~J} \mathrm{~g}^{-1}{ }^{\circ} \mathrm{C}^{-1}$

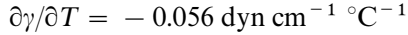

$\gamma=119.96+\left(T-T_{\mathrm{m}}\right) \partial \gamma / \partial T \mathrm{dyn}_{\mathrm{cm}}^{-1}$

$\mu=0.0302-1.533 \times 10^{-4}\left(T-T_{\mathrm{m}}\right) \mathrm{g} \mathrm{cm}^{-1} \mathrm{~s}^{-1}$

$\beta=6.6 \times 10^{-4}{ }^{\circ} \mathrm{C}^{-1}$

$\varepsilon_{\mathrm{s}}=\varepsilon_{\mathrm{L}}=0.7$

$\rho_{\mathrm{s}}=2.118 \mathrm{~g} \mathrm{~cm}^{-3}$

$\rho_{\mathrm{L}}=1.904 \mathrm{~g} \mathrm{~cm}^{-3}$

Crucible (Pyrex glass)

$\rho_{\text {cru }}=2.2 \mathrm{~g} \mathrm{~cm}^{-3}$

$C p_{\text {cru }}=0.188 \mathrm{~J} \mathrm{~g}^{-1}{ }^{\circ} \mathrm{C}^{-1}$

$k_{\text {cru }}=3.5 \times 10^{-2} \mathrm{~W} \mathrm{~cm}^{-1}{ }^{\circ} \mathrm{C}^{-1}$

$\varepsilon_{\text {cru }}=0.7$

Detailed description of the governing equations can be found in our previous reports [10]. To be self-consistent, the physical properties of $\mathrm{NaNO}_{3}$ and crucible are listed in Table 1.

The numerical solution of the governing equations was based on a FVM formulation using primitive variables and collocated grids [10], and the central difference scheme was used for both convective and diffusive fluxes. Although the firstorder upwind scheme for the convective fluxes converged slightly faster, its accuracy was poorer and thus not efficient for 3D calculations. However, the central difference was not stable for convective fluxes when they were dominant. To amend this, the deferred correction method [14] was used during iteration to retain its stability. The SIMPLE approach [15] was further used for keeping the velocity/pressure coupling.

In general, the single-grid solution was the same as before [10], but it suffered from slow convergence as the mesh was refined. The slow convergence was mainly due to the long-wavelength errors being difficult to be smoothed out by linear equation solvers. In order to accelerate the convergence, the MG schemes were used. Indeed, when the domain shape is fixed, several MG cycles can be

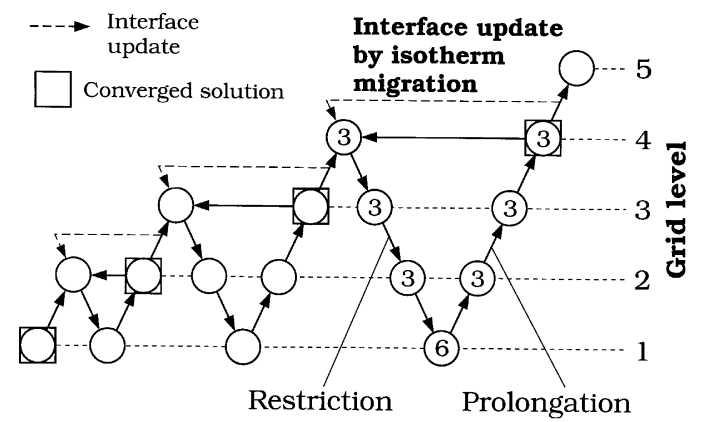

Fig. 2. Schematic of V-cycle full multigrid scheme with interface update by isotherm migration method. Only three levels of grids are used here.

used for speedup. However, through several benchmark tests, we found that the V-cycle full MG (FMG) scheme, as illustrated in Fig. 2, was effective in the computation of heat transfer and fluid flow. Now, the only problem remaining is how to update the interface shape so that the heat flux balance there can be satisfied. To do this, the isotherm migration method (IMM) was further used [10], and the interface update was performed at the top of each V-cycle of FMG, as indicated by the dashed lines in Fig. 2. In brief, during fixed-grid iterations (inner V-cycle) we removed the isotherm condition at the growth interface, while keeping the flux balance equation there for calculating the interface temperature. After the heat flow solution converged, we located the melting-point isotherm by linear interpolation. This isotherm surface was then used for grid generation. As the process continued, the interface converged, linearly, after about ten updates depending on how far away the initial interface was from the solution. Therefore, by the time of the finest grid was used, only 4-6 interface updates were needed.

The mesh layout of level two was similar to the one used before [10], and the solution had secondorder accuracy. Although it was still difficult to prove that the solution obtained was mesh independent, the flow structures for the finest two meshes were close to each other. Also, since the flow structure studied here was simple, the positions of vortices were not changed much by different meshes. 
Because the interface shape was very sensitive to the convection for $\mathrm{NaNO}_{3}$, the interface convergence usually required more than ten successive updates to reach a normalized $L_{1}$-norm of $10^{-4}$ at the first two $\mathrm{V}$ cycles. The later $\mathrm{V}$ cycles required fewer interface updates, usually $4-8$, because the final solution was approached. Unfortunately, to this system, the grid at level one needed to be fine enough to obtain a converged solution. Otherwise, the MG corrections from the coarse grids were useless. Therefore, for a case with about 0.5 million unknowns, it still took more than $2 \mathrm{~h}$ CPU time totally on an $\mathrm{HP} / \mathrm{C} 180$ workstation. Nevertheless, as compared with the single-grid approach, the FMG approach was more efficient for larger problems. In fact, the best performance was obtained for a two-phase heated box problem with all the walls being insulated, and the MG acceleration was two orders faster than the single-grid calculation. Detailed numerical calculations and performance study of the FVM/MG schemes for free-interface problems can be found in Ref. [11].

\section{Results and discussion}

At the beginning of this study, no insulation was used at the bottom of the furnace. Due to the natural convection of the surrounding air, the furnace was cooler at the bottom. As a result, the interface was extremely concave and formed a thin shell at the bottom of the crucible. Hence, singlecrystal growth was difficult. An effective way to reduce the concavity was to add an insulation at the bottom of the furnace. Fig. 3 shows the heating profiles (the upper figure) and the observed interface shape (top and side views) at different growth rates. The hot zone was kept at about $360-390^{\circ} \mathrm{C}$, while the cold zone was at $260-290^{\circ} \mathrm{C}$. The thermal gradient near the growth interface (for stationary) is about $25^{\circ} \mathrm{C} / \mathrm{cm}$. As shown, with the insulation at the bottom, near the melting point $\left(306.8^{\circ} \mathrm{C}\right)$ the lower part of the crucible becomes hotter than the top. However, the observed interfaces are all very concave. Also, as compared with the melting point of the heating profiles, the positions of the interface are very much near the cold zone. Clearly, this is caused by both buoyancy and thermocapillary
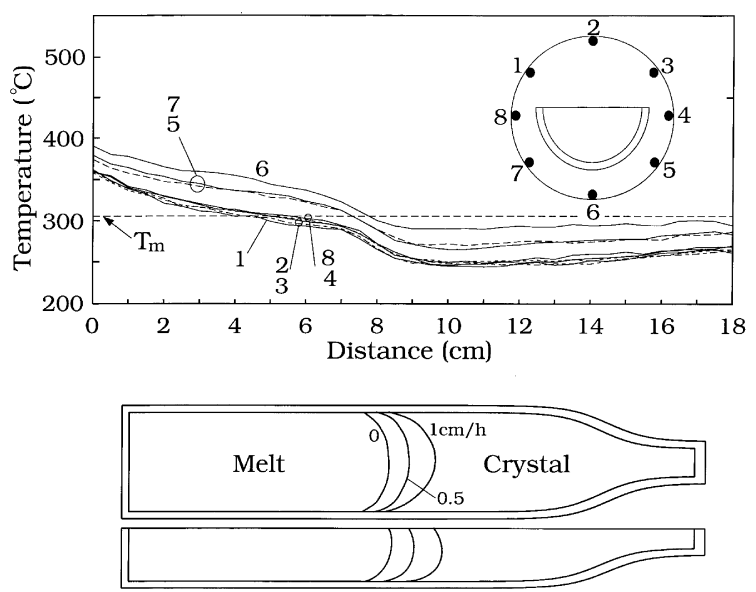

Fig. 3. Heating profiles at different positions of the inner furnace wall (on the top) and the observed interface shapes (top and side views) during steady growth at different growth rates. The bottom part of the furnace is insulated.

convections; both the flows are in clockwise direction. With the increasing growth rate, the concavity increases as a result of the release of heat fusion.

A typical observed flow pattern at the mid-plane of the melt for a case from Fig. 3 are shown in Fig. 4a; its schematic is added in Fig. 4b for illustration. Clearly, two flow cells are induced at both ends, respectively. The flow between is stretched. Such a flow pattern implies that the thermal gradients are higher at both ends, while being much smaller in the bulk melt. This can be further explained by the simulation result in Fig. 4c. As shown, the calculated flows are consistent with the observed ones. Due to the optical distortion by the melt and the crucible, the observed image is lower than the actual one, and its shape is stretched in the vertical direction. If this factor is taken into account, the agreement of the simulated result with the observed one seems to be quite good. Due to the thermocapillary effect, the velocity at the surface appears to be higher. From the distortion of the isotherms, the hot melt at the top surface is pushed towards the growth front; both thermocapillary and buoyancy flows are in the same flow direction. As a result, the interface shape is melted away from the melting point position of the heating profile. Since the isotherms are squeezed towards the growth front at the surface, the temperature 
(a)

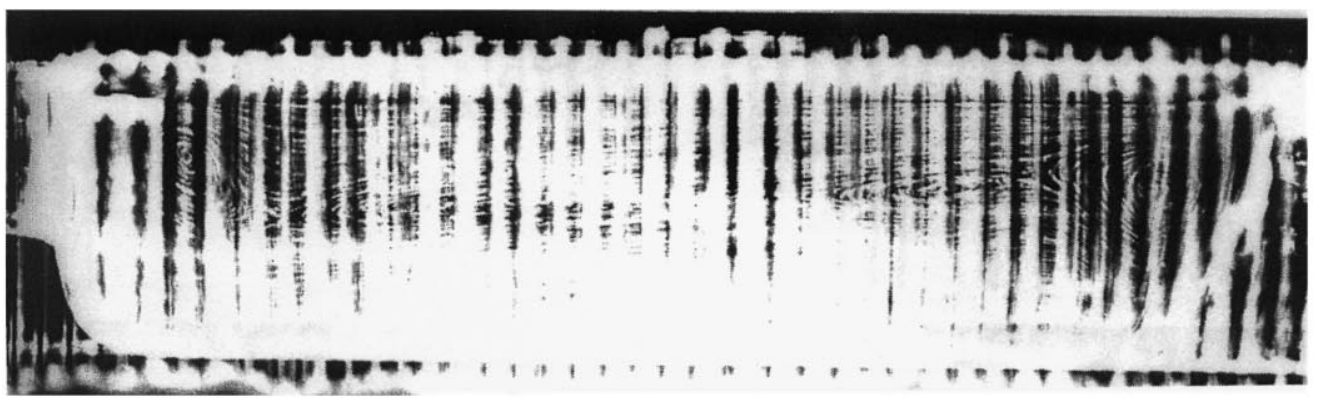

(b)

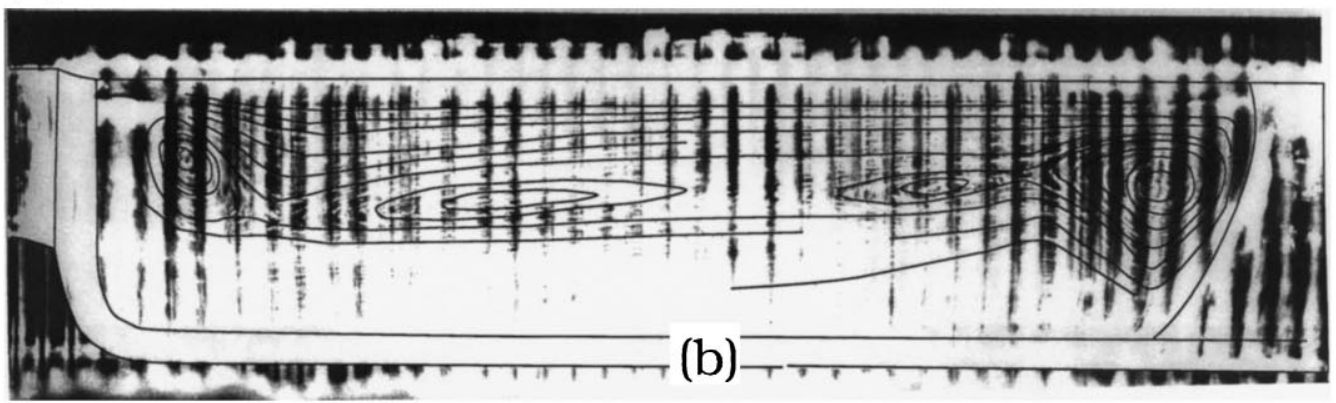

(c)

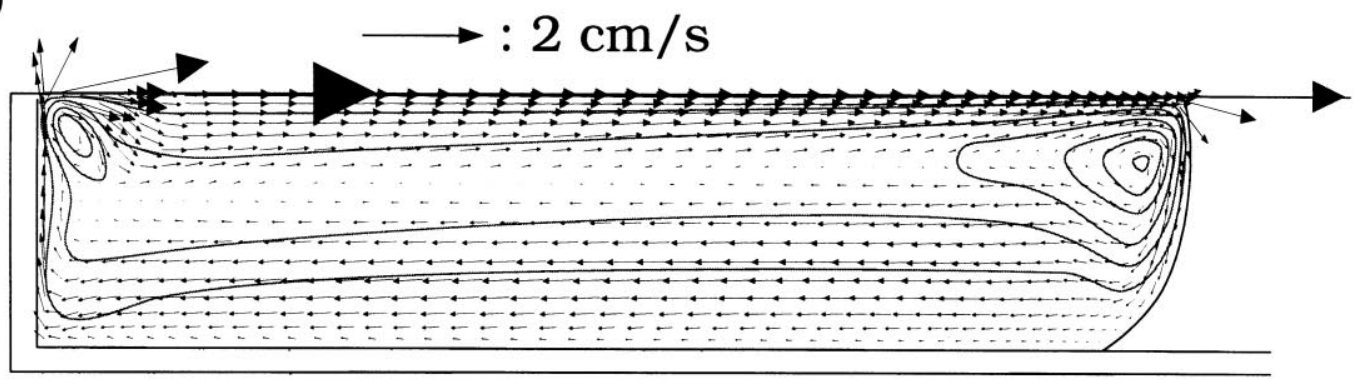

$\Delta \mathrm{T}=5^{\circ} \mathrm{C}$

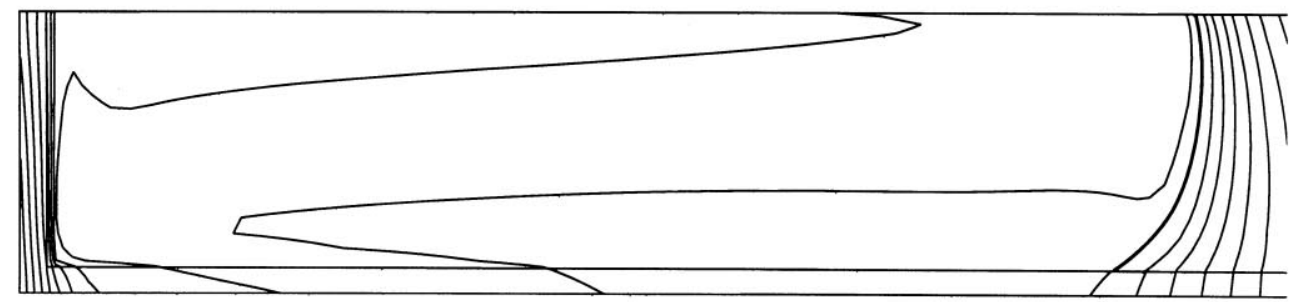

Fig. 4. (a) Observed flow patterns; (b) schematic illustration of (a); (c) calculated flow and thermal fields for (a). 
drop near the growth front is large, while in the bulk the temperature gradients are small, as shown in the bottom figure of Fig. 4c. As a result, a main flow cell is induced near the growth front. The flow cell on the left is mainly caused by the local heating from the supporting rod. To simulate this, we have purposely increased the heat transfer coefficient on the left. As illustrated, since sodium nitrate is a high Prandtl-number material, the convective heat transfer is dominant in the melt. As a result, the interface shape is significantly affected by the flow. The observed melt velocity near the surface in the bulk melt was about $3 \mathrm{~mm} / \mathrm{s}$, which also agreed well with the calculation. The top view of the simulated interface is also very concave as the observed one shown in Fig. 3, and the divergent flow near the interface due to the thermocapillary force further sharpens the interface shape near the crucible, and this deteriorates the growth. A similar observation was also found for the GaAs growth simulation [10].

It is interesting to notice that the flow pattern shown in Fig. 4a is typical for all the growth experiments here. Changing growth rates and heating profiles does not affect much the flow pattern; the observed maximum melt velocity increases with the increasing thermal gradients of the heating profiles, but the flow patterns are still similar. Computer simulation also confirms such an observation.

To reduce the concavity of the growth interface further, a typical way for Bridgman growth is to lower the cold zone temperature or to increase the thermal gradient near the interface $[10,16]$. The results are shown in Fig. 5. As shown, the cold zone is reduced to about $175^{\circ} \mathrm{C}$, while the hot zone remains the same; the thermal gradient near the growth interface is increased slightly to about $35^{\circ} \mathrm{C} / \mathrm{cm}$. As a result, the interface shapes become less concave. The effect of the growth rate on the interface, as compared with that in Fig. 3, is also significantly reduced. However, these concave interfaces are not good enough for single-crystal growth. Therefore, further improvement is necessary. From the side view of the interface, even though it is caused by the flow, keeping the bottom warmer than the top is still a right direction to reverse the interface deflection. However, at the same time we want to make the top view convex as
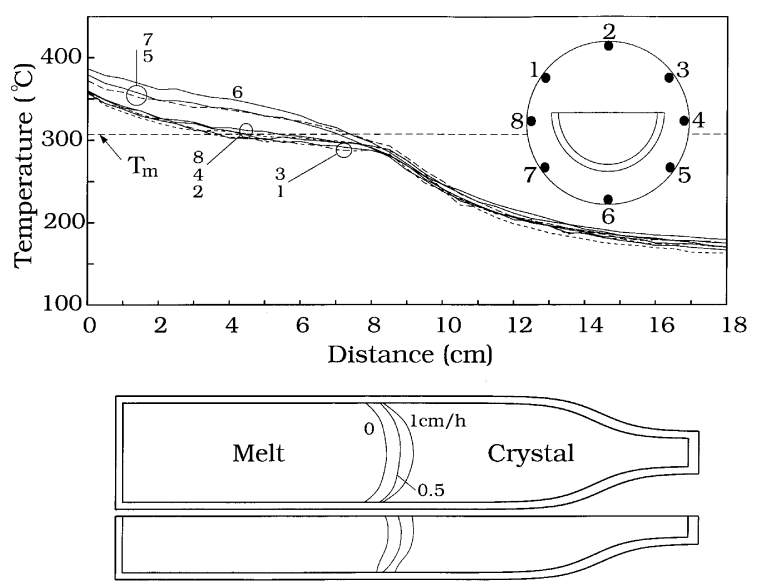

Fig. 5. Heating profiles (top) and the growth interfaces (top and side views) with higher thermal gradients near the interface. The cold zone temperature is reduced to about $175^{\circ} \mathrm{C}$.

\section{Interface}
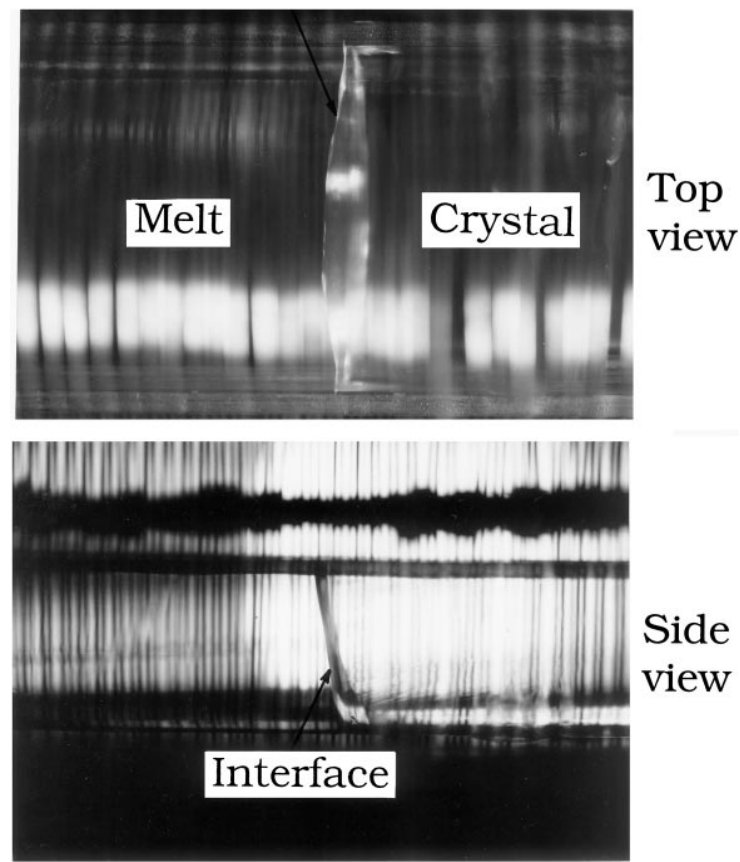

Fig. 6. Top and side views of the growth interface controlled by booster heating and top spot cooling.

well. To do this, in addition to using the booster heating near the interface, we purposely added a nozzle to blow cooling air to generate a cold spot on the furnace wall above the growth interface 

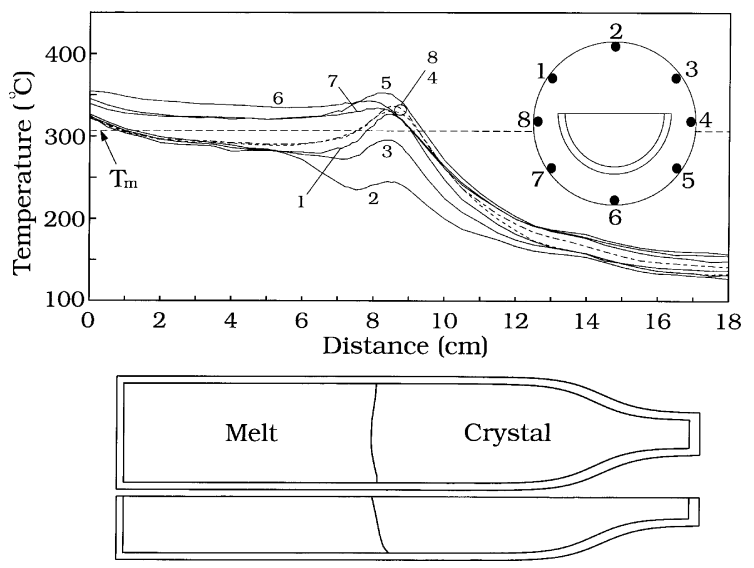

Fig. 7. Heating profiles and observed interface shapes (top and side views) for Fig. 6.

pushing the middle part of the interface towards the hot zone. With such a modification, a convex interface was obtained. The photographs of the top and side views are shown in Fig. 6. The corresponding thermal profiles are shown in Fig. 7. As shown, we have a local heating at the bottom and this makes the convex contact of the interface and the crucible. The local cooling from the top pushes the growth front beneath the cold spot towards the melt. As a result, the top view of the interface shape becomes convex. Such a growth interface allowed us to grow a single crystal easily without using a seed. Similar arrangement was also used for the zone melting configuration by using the booster heater as the main heater, while the other heaters became auxiliary ones. Again, the control of the convex interface was also easier with local spot cooling. In addition, the flow patterns for the zone melting configuration were simpler.

\section{Conclusions}

We conducted flow and interface visualization experiments during the horizontal Bridgman growth of sodium nitrate. Thermocapillary and buoyancy convections significantly affected the interface shape and also pushed it towards the cold zone significantly. The concavity of the interface shape could be reduced effectively by making the furnace hotter beneath the growth interface through a booster heater or insulation. Due to the vigorous convection, temperature was quite uniform in the bulk melt and the thermal gradients were restricted to the front of the interface inducing a major flow cell there. The left flow cell was caused by the local heating from the supporting rod. Between these two cells, the flow cell in the bulk melt was stretched, and the intensity was weak. Such a flow structure was not affected much by growth conditions. Computer simulation was also consistent with the observation. While using a booster heating for the interface, a local spot cooling at the top was found effective for interface control. The obtained convex interface shape thus improved the yield of single crystals significantly.

\section{Acknowledgements}

This work is supported by the National Science Council and the National Center for High Performance Computing of the Republic of China under Grant No. NSC87-2214-E008-008. The authors are grateful for the careful review of the referees.

\section{References}

[1] E. Monberg, in: D.T.J. Hurle (Ed.), Handbook of Crystal Growth, Vol. 2a, Basic Techniques, North-Holland, Amsterdam, 1994, p. 52.

[2] B.H.T. Chai, private communication.

[3] M.J. Crochet, F.T. Geyling, J.J. Van Schaftingen, J. Crystal Growth 65 (1983) 166.

[4] M.J. Crochet, F.T. Geyling, J.J. Van Schaftingen, Int. J. Numer. Meth. Fluids 7 (1987) 29

[5] C.W. Lan, M.K. Chen, M.C. Liang, J. Crystal Growth 187 (1998) 303.

[6] K. Edwards, J.J. Derby, J. Crystal Growth 179 (1997) 120.

[7] K. Oda, T. Saito, J. Nishihama, T. Ishihara, J. Crystal Growth 97 (1989) 186.

[8] M. Yao, H. de Groh III, Numer. Heat Transfer, Part A 24 (1993) 393.

[9] G.H. Yeoh, G. de Vahl Davis, E. Leonardi, H.C. de Groh III, M. Yao, J. Crystal Growth 173 (1997) 492.

[10] M.C. Liang, C.W. Lan, J. Crystal Growth 180 (1997) 587.

[11] C.W. Lan, M.C. Liang, J. Comput. Phys. 151 (1999) 1. 
[12] D. Schwabe, J. Metzger, J. Crystal Growth 97 (1989) 23.

[13] C.W. Lan, D.T. Yang, Numer. Heat Transfer, Part A 29 (1996) 131.

[14] P.K. Khosla, S.G. Robin, Comput. Fluids 2 (1974) 207.
[15] S.V. Patankar, Numerical Heat Transfer and Fluid Flow, Hemisphere, Washington, DC, 1980, p. 126.

[16] C.W. Lan, C.C. Ting, Int. J. Numer. Methods Heat Fluid Flow 6 (1996) 3. 